\title{
Preparation of yttrium barium copper oxide superconductive fibers via electrospinning through a lactic-acid gel route
}

\author{
Kenji IIMURA ${ }^{\dagger}$, Yuya ISHIKAWA, Takeyuki KIKUCHI, \\ Toru TAKAI, Hiroshi SATONE and Michitaka SUZUKI \\ Department of Chemical Engineering, Graduate School of Engineering, University of Hyogo, \\ 2167 Shosha Himeji, Hyogo 671-2201, Japan
}

\begin{abstract}
A new synthetic route to prepare yttrium barium copper oxide superconductive fibers using electrospinning in conjunction with the polymerizable complex method was developed. The as-spun fibers exhibited wool-like tactility and were 5-7 $\mu \mathrm{m}$ thick. The fibers therefore exhibited sufficient flexibility to be fabricated into arbitrary shapes. Although the fibers shrunk and exhibited brittle tactility because of the decomposition of organic compounds during heat treatment, the fibers predominantly maintained their fibrous form. Scanning electron microscopy observations revealed the growth of metal oxide grains during sintering. Powder X-ray diffraction pattern of the annealed fibers showed good agreement with the pattern of $\mathrm{YBa}_{2} \mathrm{Cu}_{3} \mathrm{O}_{7-\delta}$. $\mathrm{Magnetic}$ property measurements of a ground fiber sample using a superconducting quantum interference device revealed that the superconducting transition temperature of the sample was $91 \mathrm{~K}$.
\end{abstract}

(C)2017 The Ceramic Society of Japan. All rights reserved.

Key-words : YBCO, Superconducting material, Fiber, Electrospinning, Polymerizable complex method

[Received January 19, 2017; Accepted June 6, 2017]

\section{Introduction}

Since the initial discovery by Onnes in 1911 that the electrical conductivity of mercury suddenly falls to zero at $4.2 \mathrm{~K}$ or below, numerous superconducting materials have been reported. Among them, metal oxide superconducting materials are considered promising because they exhibit high superconducting transition temperatures $T_{\mathrm{c}}$, enabling liquid nitrogen to be used as the coolant. Among high-temperature superconducting materials, yttrium barium copper oxide (YBCO) is quite promising because of its excellent properties under high magnetic fields. ${ }^{1)}$ In terms of the practical applications of metal oxide superconducting materials, fibers are a notable ${ }^{2)}$ form because heat transfer during cooling will likely be enhanced because of their large interface with the coolant. The presence of certain conductive paths is also expected because of fibers' one-dimensional structure. The most promising property of fibrous superconductors is likely their flexibility, which enables them to be molded into arbitrary shapes or to be used to fabricate electrical circuits. However, the fabrication of metal oxides into fibrous form is generally difficult because they are too brittle. Therefore, a new approach has been sought to obtain a fibrous metal oxide superconducting material directly. Sol-gel synthesis is a typical technique for preparing films or fibers; however, a strategy based on the hydrolysis and condensation of alkoxides ${ }^{3)}$ is complicated, especially in case of a multicomponent system, and the chemical substances involved are expensive. Furthermore, such a system must be essentially inhomogeneous because different components naturally have different hydrolysis rates, which often results in precipitation. An acetate-based system has been also reported as a low-cost synthesis method; ${ }^{4)-6)}$ however, the method used to obtain a stable solution during the preparation process is complex.

Corresponding author: K. Iimura; E-mail: iimura@eng.u-hyogo. ac.jp
To overcome these problems, we focused on the polymerizable complex method ${ }^{7), 8)}$ to prepare a spinning solution for a subsequent electrospinning process. In the polymerizable complex method, metals are dissolved in a solvent, forming a stable complex and resulting in a homogeneous solution; the metals are then immobilized by polymerization of the complexing agent and solvent. This process avoids the segregation of metal species, and the subsequent combustion or decomposition of the polymer provides homogeneous residual metal oxides. In the most commonly used process, citric acid is used as the complexing agent and ethylene glycol is used as the polymerizable solvent. Citric acid has three carboxyl groups and a hydroxyl group, thus forming a quite stable chelate complex. In addition, excess hydroxyl groups can react with ethylene glycol to form ester bonds via heating. Thus, a polyester resin containing metal species with the desired atomic ratio can be obtained. However, the most important point is that the mass ratio between organic substances and metals is large (typically eight or more); such a large amount of organic matter makes maintaining the original form of a solid, film or fiber before the delipidation process difficult. In addition, the isotropic three-dimensional network molecular structure of the aforementioned polyester is not suitable for spinning.

To obtain fibrous YBCO, we developed a new system using lactic acid as both a chelating agent and a polymerizable solvent. Lactic acid has been reported to form a chelate complex with specific ions such as titanium; ; ${ }^{9}$ thus, chelation, or at least strong interactions, with ionic species is expected in our system. Because lactic acid molecules include both a carboxyl group and a hydroxyl group, they can polymerize without any other substance such as a glycol. This feature can be exploited to reduce the amount of organic matter in the system. In the system adopted here, lactic acid forms a complex or strongly interacts with metal species, then undergoes polymerization to polylactic acid via heating. Notably, the mass ratio of organic matter is reduced to approximately two and the polylactic acid exhibits a ladder-like, 
one-dimensional structure, making it suitable for spinning. Duarte et al. ${ }^{10)}$ have reported the fabrication of YBCO nanofibers using approaches similar to those in the present work, where polyvinylpyrrolidone (PVP) was used as an auxiliary agent for spinning. However, the fibers obtained with a water-soluble polymer such as PVP at low content exhibited a small diameter and were too soft to sustain any structural form. Thus, the fibers fall and pile up, forming thin sheets, which limits the applications of this material. In the present paper, a procedure used to prepare the spinning solution and to carry out the electrospinning to obtain YBCO fibers with sufficient mechanical strength to be formed into an arbitrary shape is reported; the characteristics of the obtained fiber are subsequently demonstrated.

\section{Experiments}

\subsection{Preparation of spinning solution}

The content of chemical substances is listed in Table 1, where the molar ratios are based on the amount of yttrium acetate tetrahydrate. The yttrium acetate tetrahydrate was purchased from Mitsuwa Chemicals; other reagents were purchased from Kishida Chemical. All reagents were analytical grade and were used without further purification. Five-sixths of the total mass of propionic acid was poured into a beaker and heated to $70^{\circ} \mathrm{C}$ using a stirrer/hotplate. The designated amounts of yttrium acetate, barium acetate, and copper(II) acetate monohydrate were dissolved and added, in this stated order, to the propionic acid under vigorous stirring to avoid evaporating the propionic acid. Propionic acid was used as a solvent for metallic salts in this work because it is a better solvent than acetic acid; thus, less propionic acid is necessary compared to the amount of acetic acid needed, thereby reducing the amount of residual organic species. Notably, the metal acetates were added to the solution after they were completely dissolved. In addition, because the solution became dark-blue after the addition of copper(II) acetate monohydrate, which made observation of the extent of dissolution difficult, mixing was continued for $1 \mathrm{~h}$ after the copper(II) acetate had been added. The designated amount of lactic acid was dissolved into the remaining one-sixth of the total mass of propionic acid, and this solution was added to the aforementioned acetate solution. After the addition of lactic acid, the resultant mixture was transferred to a flask and then stirred under an open system environment in an oil bath at $100^{\circ} \mathrm{C}$. Because of the evaporation of propionic acid and the polymerization of lactic acid, the viscosity of the solution increased. Stirring was continued until the viscosity of the solution reached $3 \mathrm{~Pa} \cdot \mathrm{s}$. The viscosity was measured using an oscillating viscometer (CBC, VISCOMATE VM-10A).

\subsection{Fundamentals of electrospinning and process parameters ${ }^{11), 12)}$}

Figure 1 shows a schematic of the electrospinning setup. Although a syringe pump is often used in such setups to control the flow rate of the spinning solution, a pump was not used in this work; instead, the spinning solution flowed through the tip of a 1.5-mm-diameter syringe under gravitational force. A high voltage was directly supplied to the solution via a wire electrode immersed in the solution. In this study, the tip-collector distance was fixed at $200 \mathrm{~mm}$ and the applied voltage, which was provided by a high-voltage power supply (Pulse Electronic Engineering, model 502), was fixed at $20 \mathrm{kV}$.

\subsection{Characterization}

Thermogravimetry-differential thermal analysis (TG/DTA) was conducted using a Rigaku TG8120 to determine the appropriate heat-treatment conditions for the obtained fibers. The measurements were carried out in air from room temperature to $1000^{\circ} \mathrm{C}$ at a heating rate of $5^{\circ} \mathrm{C} / \mathrm{min}$. According to the results of TG/DTA measurements (detailed later), the appropriate heattreatment conditions were as follows. Fibers were first heated using an electric furnace (AS ONE, MMF-1) in air from room temperature to $600^{\circ} \mathrm{C}$ at a heating rate if $10^{\circ} \mathrm{C} / \mathrm{h}$ for calcination; the temperature was maintained at $600^{\circ} \mathrm{C}$ for $5 \mathrm{~h}$, and the sample was then cooled to room temperature in the furnace. The fibers were then heated using the same electric furnace from room temperature to $920^{\circ} \mathrm{C}$ at a heating rate of $10^{\circ} \mathrm{C} / \mathrm{h}$ for sintering. The temperature was maintained at $920^{\circ} \mathrm{C}$ for $5 \mathrm{~h}$, and the sample was again cooled to room temperature in the furnace. Finally, the fibers were heated from room temperature to $500^{\circ} \mathrm{C}$ at a heating rate of $10^{\circ} \mathrm{C} / \mathrm{h}$ using a tube furnace (Koyo Thermo Systems, $\mathrm{KTF} 035 \mathrm{~N}$ ) under an $\mathrm{O}_{2}$ atmosphere at ambient pressure for annealing; the $\mathrm{O}_{2}$ gas flow rate was $0.4 \mathrm{~L} / \mathrm{min}$.

The heat-treated fibers were characterized by powder X-ray diffraction (XRD) on a diffractometer (Rigaku, Mini Flex II) equipped with a $\mathrm{Cu}-\mathrm{K} \alpha$ radiation source; the scan rate was

Table 1. Molar ratio of chemical substances normalized to the amount of yttrium acetate monohydrate

\begin{tabular}{lccccc}
\hline Chemical substance & $\mathrm{Y}\left(\mathrm{CH}_{3} \mathrm{COO}\right)_{3} \cdot 4 \mathrm{H}_{2} \mathrm{O}$ & $\mathrm{Ba}\left(\mathrm{CH}_{3} \mathrm{COO}\right)_{2}$ & $\mathrm{Cu}\left(\mathrm{CH}_{3} \mathrm{COO}\right)_{2} \cdot \mathrm{H}_{2} \mathrm{O}$ & $\mathrm{CH}_{3} \mathrm{CH}_{2} \mathrm{COOH}$ & $\mathrm{CH} \mathrm{CH}_{2}(\mathrm{OH}) \mathrm{COOH}$ \\
\hline Molar ratio & 1.0 & 2.0 & 3.0 & 0.08 & 6.0 \\
\hline
\end{tabular}

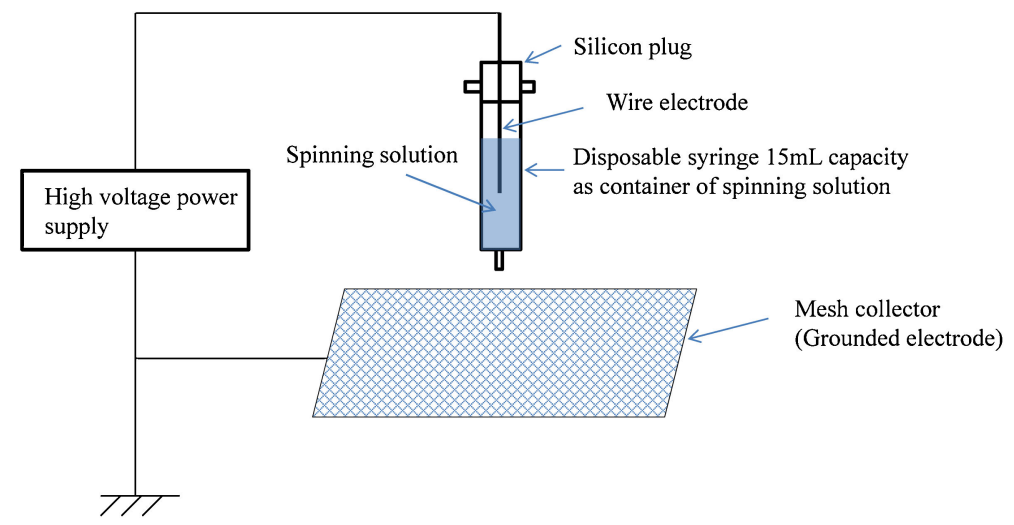

Fig. 1. Schematic of the electrospinning setup. 
$2.0^{\circ} / \mathrm{min}$, the step interval was $0.02^{\circ}$, and the scan range was $10-70^{\circ}$ in $2 \theta$. Scanning electron microscopy (SEM) (Keyence, VE-8800) was used to observe the fiber morphology. Magnetic properties were characterized using a Quantum Design MPMS2 equipped with a superconducting quantum interference device (SQUID) to determine the $T_{\mathrm{c}}$.

\section{Results and discussion}

Figure 2(a) shows a digital image of fibers obtained via electrospinning. The fibers clearly exhibited wool-like tactility. Although the color of the spinning solution was dark-blue, the obtained fibers exhibited a light-blue color because of the scattering of visible light resulting from the fineness of the fibers. The SEM image in Fig. 2(b) shows that the fiber diameter ranged from 5 to $7 \mu \mathrm{m}$ and that the surface was quite smooth, with no precipitates of metal species; these observations suggest that the metal species in the fibers were uniformly distributed. The obtained fibers exhibited sufficient flexibility and strength to be formed as a nonwoven fiber mat with a felting needle, as demonstrated in Fig. 3. This flexibility suggests that these fibers may have unprecedented new applications. In addition, films can be obtained easily altering the process parameters such as the viscosity of the spinning solution.

Figure 4 shows the results of TG/DTA measurements. The observed weight loss as the temperature was increased to $200^{\circ} \mathrm{C}$ was likely due to the evaporation of unreacted propionic acid and lactic acid. The rapid decrease of weight at $210^{\circ} \mathrm{C}$, which was accompanied by an exothermic DTA peak, suggests the combustion of the polymer. The slight weight loss beginning at approximately $800^{\circ} \mathrm{C}$ is plausibly due to the formation of the $\mathrm{YBCO}$
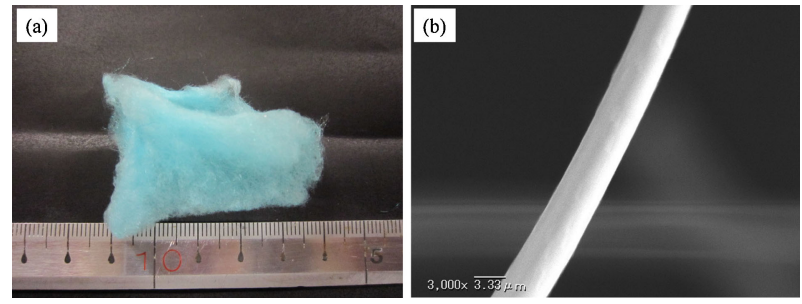

Fig. 2. Digital image (a) and SEM image (b) of the obtained fibers.
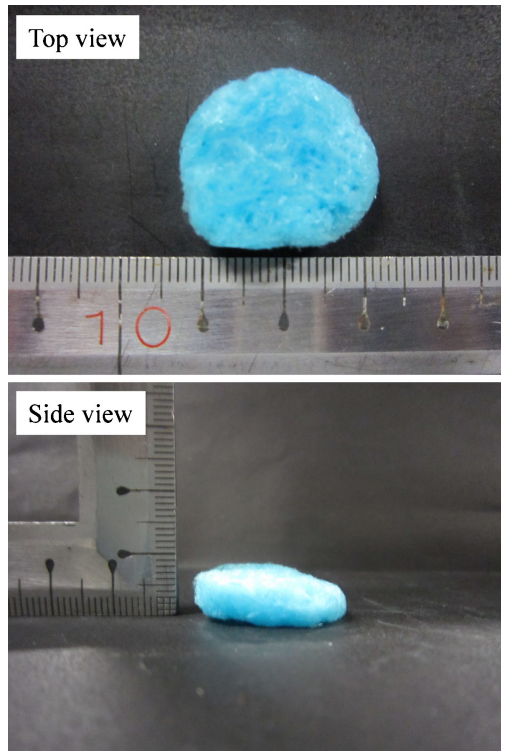

Fig. 3. Digital image of the fabricated nonwoven mat. phase. Notably, the total weight loss was approximately $55 \%$; this small amount of organic matter led us to expectation that the fibers would maintain their fibrous form when subjected to heat treatment.

Figures 5(a) and 5(b) show a digital image and an SEM image, respectively, of the fibers after calcination. The digital image clearly shows that the fibers' color changed to black; however, the fibers still maintained their wool-like form. The SEM image reveals that the morphology of the fibers did not change drastically although their surface became rough with numerous small bumps. Figures 6(a) and 6(b) show a digital image and an SEM image, respectively, of the sintered fibers. Although the fibers became brittle to a large extent, as demonstrated by the observation of fragments in Fig. 6(a), they

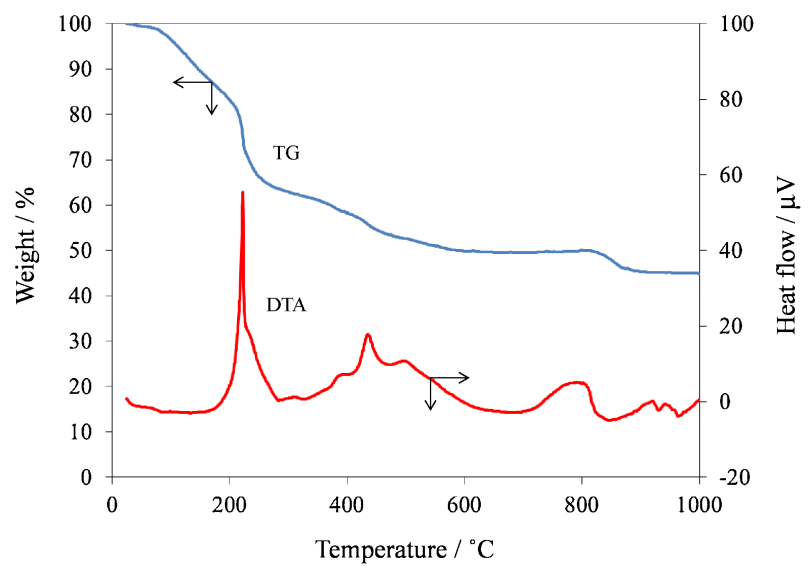

Fig. 4. TG/DTA profile of the obtained fibers during heat treatment in air.
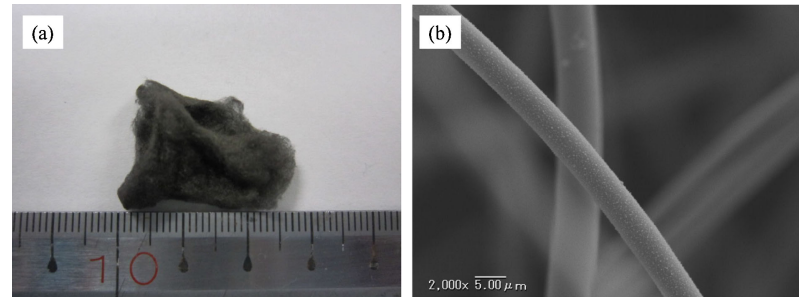

Fig. 5. Digital image (a) and SEM image (b) of the fibers after calcination.
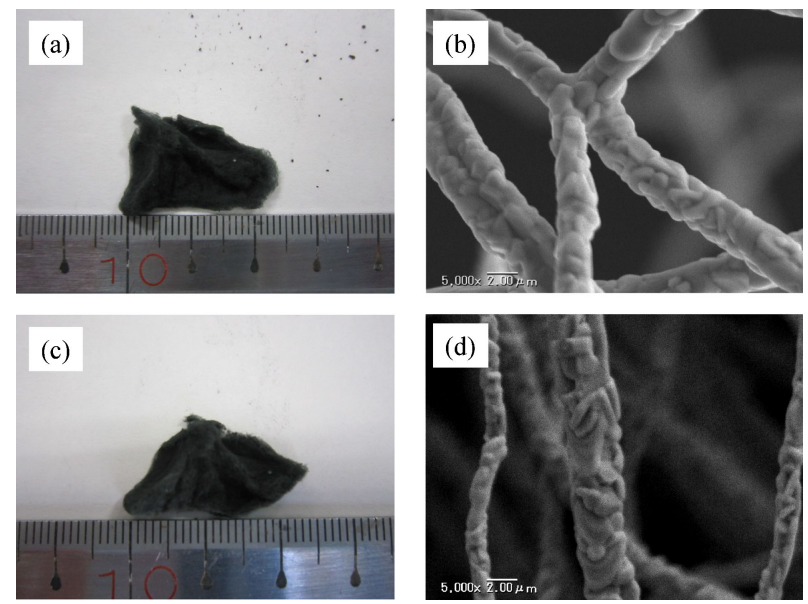

Fig. 6. Digital images (a, b) and SEM images (c, d) of fibers after sintering and annealing, respectively. 


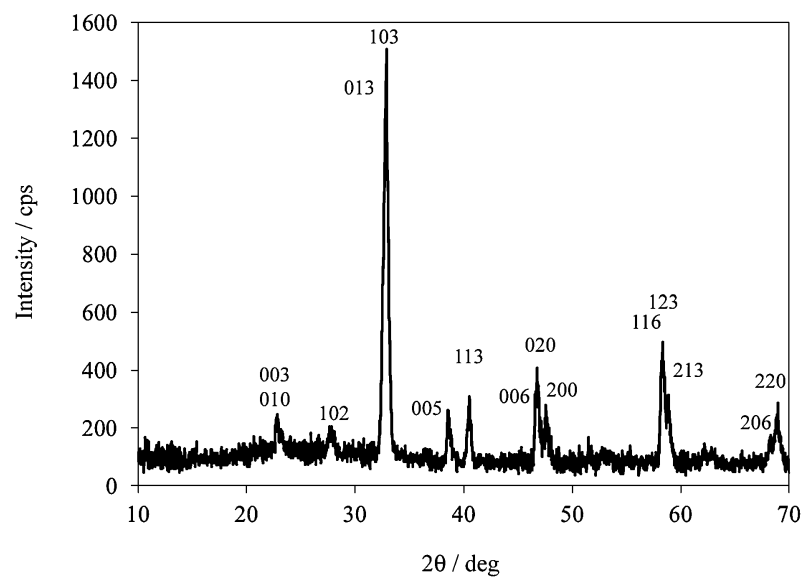

Fig. 7. XRD pattern of the annealed fiber sample.

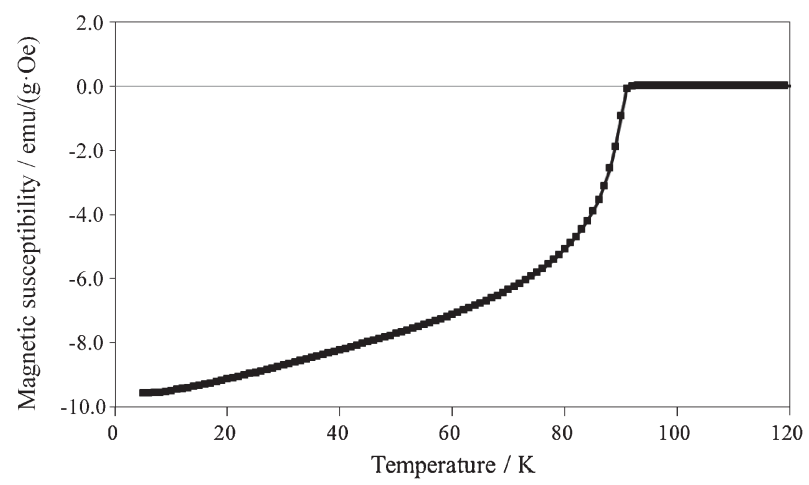

Fig. 8. Magnetic susceptibility of the annealed fiber sample as a function of temperature.

maintained their fibrous structure. The morphology of the fibers drastically changed, as evident in Fig. 6(b), where grains of metal oxides grew, making the surface quite rough. Figures 6(c) and 6(d) show a digital image and an SEM image, respectively, of the annealed fibers. No drastic change in appearance from that of the sintered sample was observed; however, further growth of grains was evident.

Figure 7 shows the XRD pattern of the annealed sample, where the fibers were ground using a mortar. The observed diffraction peaks were indexed to the unit cell of $\mathrm{YBa}_{2} \mathrm{Cu}_{3} \mathrm{O}_{7-\delta}$, which is a well-defined typical superconducting phase. Figure 8 shows the results of magnetic property measurements, where the zero-field-cooled magnetic susceptibility is plotted as a function of temperature. The sample clearly exhibits diamagnetic behavior at temperatures below $91 \mathrm{~K}$. As a result, the $T_{\mathrm{c}}$ of the sample prepared in this work was confirmed to be $91 \mathrm{~K}$. Notably, the fibers were also ground for the magnetic property measurement; thus, the magnetic properties of the fibers in fibrous form remain unclear. This topic is left for future work.

\section{Conclusion}

A new synthetic route to YBCO superconductive fibers using electrospinning together with the polymerizable complex method was successfully developed. The tactile, as-spun fibers were

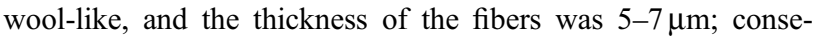
quently, the fibers exhibited sufficient flexibility to be fabricated into arbitrary shapes. The results of TG/DTA measurements revealed that the mass fraction of organic substance was reduced to $55 \%$, which is remarkably low compared with the corresponding fraction in fibers prepared by the conventional polymerizable complex method. Although the fibers shrunk and exhibited brittle tactility because of the decomposition of organic substances during the heat treatment, the fibers nonetheless predominantly maintained their fibrous form because of their reduced content of organic matter. SEM observations revealed the growth of metal oxide grains during sintering. The powder XRD pattern of the annealed fibers were consistent with the pattern of $\mathrm{YBa}_{2} \mathrm{Cu}_{3} \mathrm{O}_{7-\delta}$. The magnetic property measurements using a SQUID magnetometer revealed that the ground samples exhibited a $T_{\mathrm{c}}$ of $91 \mathrm{~K}$.

\section{References}

1) P. H. Hor, R. L. Meng, L. Gao, Z. J. Huang, Y. Q. Wang and C. W. Chy, Phys. Rev. Lett., 58, 908-910 (1987).

2) T. Goto and M. Tsujihara, J. Mater. Sci. Lett., 7, 283-284 (1988).

3) Y. Masuda, T. Takeishi, K. Matsubara, R. Ogawa and Y. Kawate, Jpn. J. Appl. Phys., 30, 1390-1397 (1991).

4) P. C. McIntyre, M. J. Cima and A. Roshko, J. Appl. Phys., 77, 5263-5272 (1995).

5) Y. Chen, G. Zhao, L. Lei and X. Liu, Supercond. Sci. Tech., 20, 251-255 (2007).

6) L. F. Admaimai, L. Daza, P. Grange and B. Delmon, J. Mater. Sci. Lett., 13, 668-670 (1994).

7) M. Kakihana, L. Börjesson, S. Eriksson and P. Svedlindh, J. Appl. Phys., 69, 867-873 (1991).

8) M. Kakihana, J. Ceram. Soc. Jpn., 117, 857-862 (2009).

9) M. Kakihana, K. Tomita, V. Petrykin, M. Tada, S. Sasaki and Y. Nakamura, Inorg. Chem., 43, 4546-4548 (2004).

10) E. A. Duarte, N. G. Rudawski, P. A. Quintero, M. W. Meisel and J. C. Nino, Supercond. Sci. Tech., 28, 015006 (2015).

11) K. Iimura, T. Oi, M. Suzuki and M. Hirota, Adv. Powder Technol., 21, 64-68 (2010).

12) K. Iimura, T. Oi, T. Kikuchi, H. Satone and M. Suzuki, J. Ceram. Soc. Jpn., 122, 349-353 (2014). 